\title{
RXRa provokes tumor suppression through p53/p21/p16 and PI3K-AKT signaling pathways during stem cell differentiation and in cancer cells
}

\author{
Rui Zhang ${ }^{1}$, Hui Li', Shuangshuang Zhang ${ }^{1}$, Yujie Zhang ${ }^{1}$, Nan Wang ${ }^{1}$, Hao Zhou', Hongpeng He', Guang Hu', \\ Tong-Cun Zhang ${ }^{1,2}$ and Wenjian Ma ${ }^{1,3}$
}

\begin{abstract}
The retinoid $X$ receptor alpha ( $R X R a)$ is an important therapeutic target impacting diverse biological processes. Activation of RXRa is known to suppress cancer cell growth. However, the cellular mechanism has been elusive. In the present study, we addressed its role during stem cell differentiation and the underlying connections with carcinogenesis. RXRa was significantly upregulated following the differentiation of human mesenchymal stem cell (hMSC) toward the formation of endothelial cell (EC). However, overexpression of RXRa in hMSC provoked a senescence-like phenotype accompanied by the elevation of tumor suppressor p53, p21, and p16. Consistently, RXRa level was suppressed in cancer cells ( five times lower compared to differentiated hMSC), and its elevation could inhibit the proliferation, migration, and angiogenesis of cancer cells. We further demonstrated that these inhibitory effects were related to RXRa's interaction with estrogen receptor a (ERa) as well as EGF and ANGPTL3 through modulating PI3K/AKT signaling pathway by AKT and FAK phosphorylation. Moreover, RXRa inhibited glycolytic metabolism in cancer cells, which might be underlying its inhibition of differentiation and carcinogenic features. These data suggest that RXRa acts as a suppressor rather than a driving force during stem cell differentiation, and unbalanced RXRa can trigger multiple yet connected signaling pathways in preventing carcinogenesis.
\end{abstract}

\section{Introduction}

Cancer cells and stem cells share similarities, such as the ability of self-renewal and the potential for differentiation ${ }^{1}$. It has been proposed that cancer cells might be originated from certain stem cells with malignant mutations termed cancer stem cells $(\mathrm{CSCs})^{2,3}$. CSCs showed higher resistance to various commonly used chemotherapeutic

\footnotetext{
Correspondence: Tong-Cun Zhang (zhangtongcun@wust.edu.cn) or Wenjian Ma (ma_wj@tust.edu.cn)

${ }^{1}$ College of Biotechnology, Tianjin University of Science and Technology, 300457 Tianjin, China

${ }^{2}$ Institute of Biology and Medicine, Wuhan University of Science and Technology, 430081 Wuhan, China

Full list of author information is available at the end of the article.

Edited by Y. Haupt
}

treatments ${ }^{4-7}$, and are believed to be a driving force for tumor recurrence and metastasis ${ }^{8-10}$.

The multistep process of cancer progression requires genome alterations that accumulated with cell proliferations and divisions ${ }^{1}$. The occurrence rate is low in normal cells owing to the limited number of cell divisions. However, the probability of accumulating multiple mutations in stem cells could be greatly elevated with their unlimited dividing capacity'. Tomasetti et al. reported recently that the occurrence of cancer is strongly correlated with the number of stem cell divisions in different tissues, which extended over five orders of magnitude based on the analysis of 31 cancer types $^{11}$. This provided a strong support to the cancer stem cell hypothesis and emphasized the importance of cell division during carcinogenesis.

\section{(c) The Author(s) 2018}

(c) (i) Open Access This article is licensed under a Creative Commons Attribution 4.0 International License, which permits use, sharing, adaptation, distribution and reproduction in any medium or format, as long as you give appropriate credit to the original author(s) and the source, provide a link to the Creative Commons license, and indicate if changes were made. The images or other third party material in this article are included in the article's Creative Commons license, unless indicated otherwise in a credit line to the material. If material is not included in the article's Creative Commons license and your intended use is not permitted by statutory regulation or exceeds the permitted use, you will need to obtain permission directly from the copyright holder. To view a copy of this license, visit http://creativecommons.org/licenses/by/4.0/. 

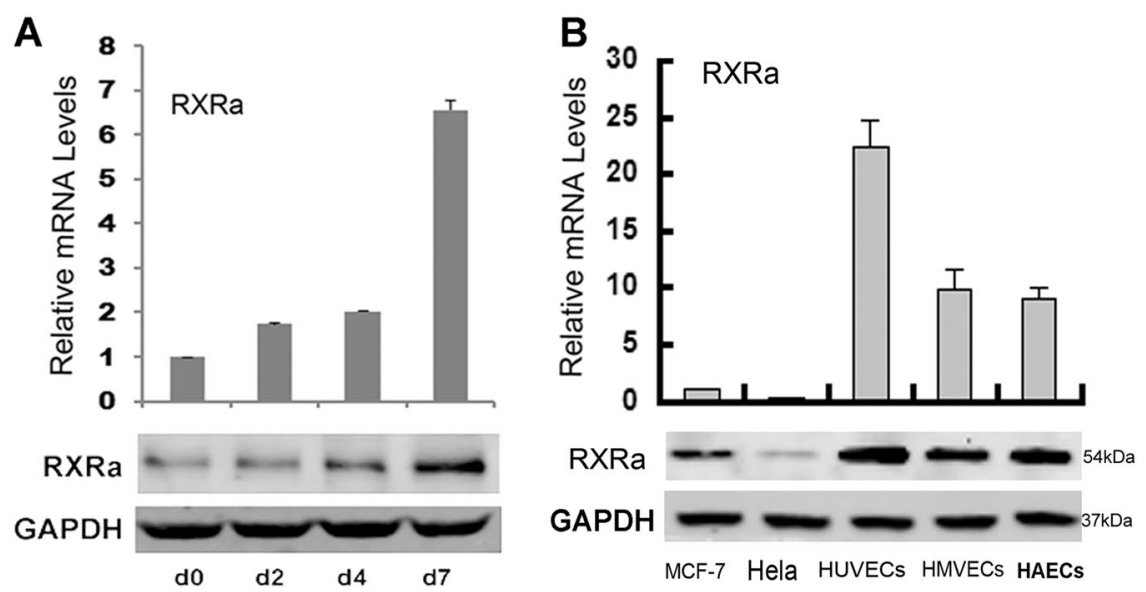

Fig. 1 RXRa expression during differentiation of hMSCs in normal epithelial cells and cancer cells. a The expression of RXRa in hMSCS cultured in EDM at days 0, 2, 4, and 7. $\mathbf{b}$ The expression of RXRa in cancer cells MCF-7 and HeLa, endothelial cells HUVECs, HMVECs, and HAVECs. The expression was determined by qRT-PCR and western blotting $(P<0.01, n=3)$

Considering that differentiated cells rarely proliferate, modulation of the cellular mechanisms to prevent stem cells from differentiation but retain at certain stages with proliferation capacity might be required in order to obtain sufficient genetic alterations for carcinogenesis. The cross talk between stem cell differentiation and carcinogenesis has been largely unknown. It is interesting to find out whether modulating stem cell differentiation could facilitate the conversion of normal stem cells into CSCs.

In the present study, we have addressed the role of retinoic acid receptor $\alpha(\mathrm{RXR} \alpha)$ in attempting to identify the cellular components that may impact both stem cell differentiation and neoplastic transformation. RXR is a family of nuclear receptors implicated in the control of a variety of physiological processes such as lipid and glucose metabolism and immune responses ${ }^{12,13}$. Some RXR isoforms have even been shown that can facilitate the induction of pluripotent stem cells $^{14,15}$. Being the most abundant and functional isoform of RXR in various cell types, RXR $\alpha$ is a central transcriptional regulator in modulating gene expression by heterodimerization with other nuclear receptors ${ }^{16}$.

Regulation of RXR by natural and synthetic ligands (e.g., vitamin $\mathrm{A}$ and retinoic acid derivatives) is known to inhibit cell proliferation and has been used to treat cancers ${ }^{17-19}$. However, the underlying mechanism is not fully understood. Here, using human mesenchymal stem cells (hMSC) as a model for stem cell differentiation, and by comparing with cancer cell lines, we sought to determine the cellular consequences of modulating RXR $\alpha$ during cell differentiation as well as the possible connections with carcinogenesis.

\section{Results}

RXRa was increasingly expressed during the differentiation of hMSC into epithelial cells but was generally suppressed in cancer cells

Tumor progression requires the activation of an "angiogenic switch" to drive the formation of new vessels, which involves the formation of new endothelial cells ${ }^{20}$. Endothelial cells can be differentiated from hMSCs, and it has been used for adult vascular repair and regeneration therapies ${ }^{21}$. To investigate what role RXR $\alpha$ plays during this process, we first determined the expression of RXR $\alpha$ during the differentiation of hMSCs toward endothelial cells. As shown in Figure 1a, RXR $\alpha$ protein level was increased in a time-dependent manner during differentiation, showing a sharp increase ( seven fold) at day 7 when endothelial cells were formed. In contrast, the RXR $\alpha$ levels determined in various human cancer cell lines were much lower. Of eight cancer cell lines that were tested (HeLa and MCF-7 were shown in Figure 1 as representatives), RXR $\alpha$ levels were found to be 5-20 times lower than that in various endothelial cell lines (HUVECs, HMVECs, and HAVECs) that hMSC can differentiate into as well as in the non-transformed breast cell line MCF10a (used as control for MCF-7) (Fig. 1b and Supplemental Fig. S1). These data on the one hand confirmed that RXR $\alpha$ played an important role during cell differentiation. On the other hand, it raises an interesting question-does suppressing RXR $\alpha$ during stem cell differentiation facilitates the process of carcinogenesis, which may account for its low expression level in cancer cells? 


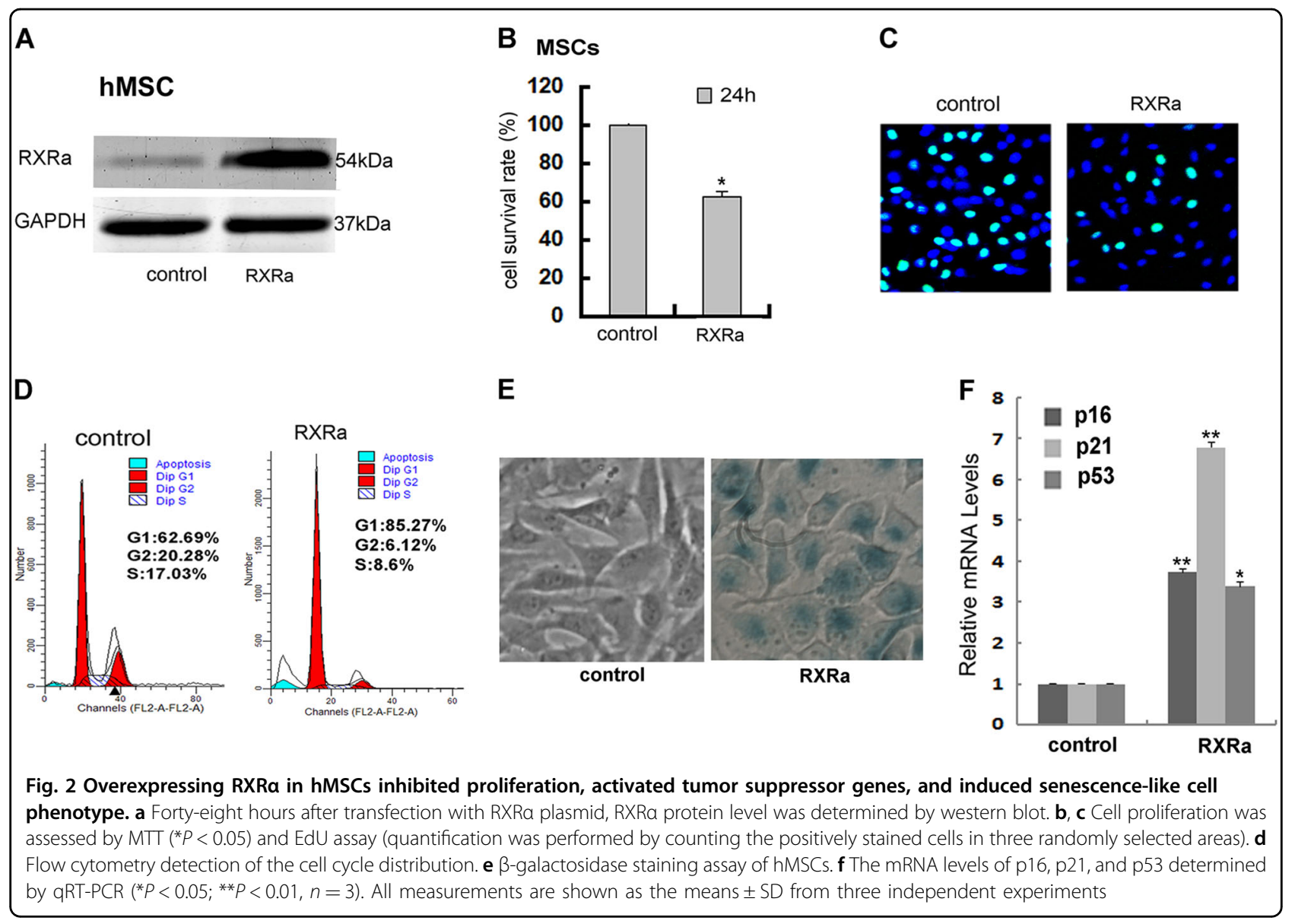

Overexpression of RXRa in hMSCs inhibited proliferation, activated tumor suppressor genes, and induced senescence-like cell phenotype

Since RXR $\alpha$ was expressed at a relatively low level in hMSCs compared to differentiated cells, we first asked whether ectopic overexpression of RXR $\alpha$ in hMSCs would speed up the differentiation process. Interestingly, overexpressing RXR $\alpha$ in hMSCs (nine times increase compared to the basal level of non-transfected control cells, Fig. 2a) did not elevate the differentiation process. Instead, it induced phenotypic changes reminiscent of cellular senescence. As determined by MTT and EdU analysis, hMSCs transfected with RXR $\alpha$ exhibited a decrease in cell growth rate (Fig. $2 \mathrm{~b}-\mathrm{c}$ ). The proportion of cells in G0/G1 phases was significantly increased and the proportion of cells in $\mathrm{S}$ phase was decreased. These data suggest that RXR $\alpha$ overexpression in hMSCs inhibited proliferation by inducing cell cycle arrest in the G0/G1 phase (Fig. 2d). In addition, positive staining of the senescence-associated $\beta$ galactosidase, a widely used marker for cellular senescence $^{22,23}$, was observed in hMSCs after transfection with RXR $\alpha$ (Fig. 2e). Furthermore, the expression of major tumor suppressor genes p53, p21, and p16 were all induced in hMSCs when RXR $\alpha$ was overexpressed, especially the level of $\mathrm{p} 21$, which was increased by seven times (Fig. 2f). Overall, these results indicate that the elevated level of RXR $\alpha$ observed during the differentiation of hMSCs is not a driving force facilitating the progression of differentiation. In fact, it negatively controls cell proliferation, and overexpression might be even harmful to cells at an early stage of cell differentiation.

\section{RXRa overexpression in cancer cells inhibited cell proliferation, invasion, and angiogenesis}

To understand why RXR $\alpha$ was suppressed in cancer cells, we overexpressed it in MCF-7 cells. Consistent with the activation of tumor-suppressing mechanism described above, overexpression of $\operatorname{RXR} \alpha$ showed some inhibitory effects on cell proliferation, migration, and tube formation capacity as described below.

The MTT and EdU data showed that RXR $\alpha$ overexpression exhibited a decrease in cell growth rate in MCF-7 (Fig. 3a-b). The proportion of cells at G0/G1 phases was evidently increased while the proportion of $S$ phase cells was decreased, suggesting that RXR $\alpha$ overexpression also inhibited proliferation by inducing cell cycle arrest in G0/G1 phase (Fig. 3c). Unlike the transient overexpression of RXR $\alpha$ investigated in the present study, 


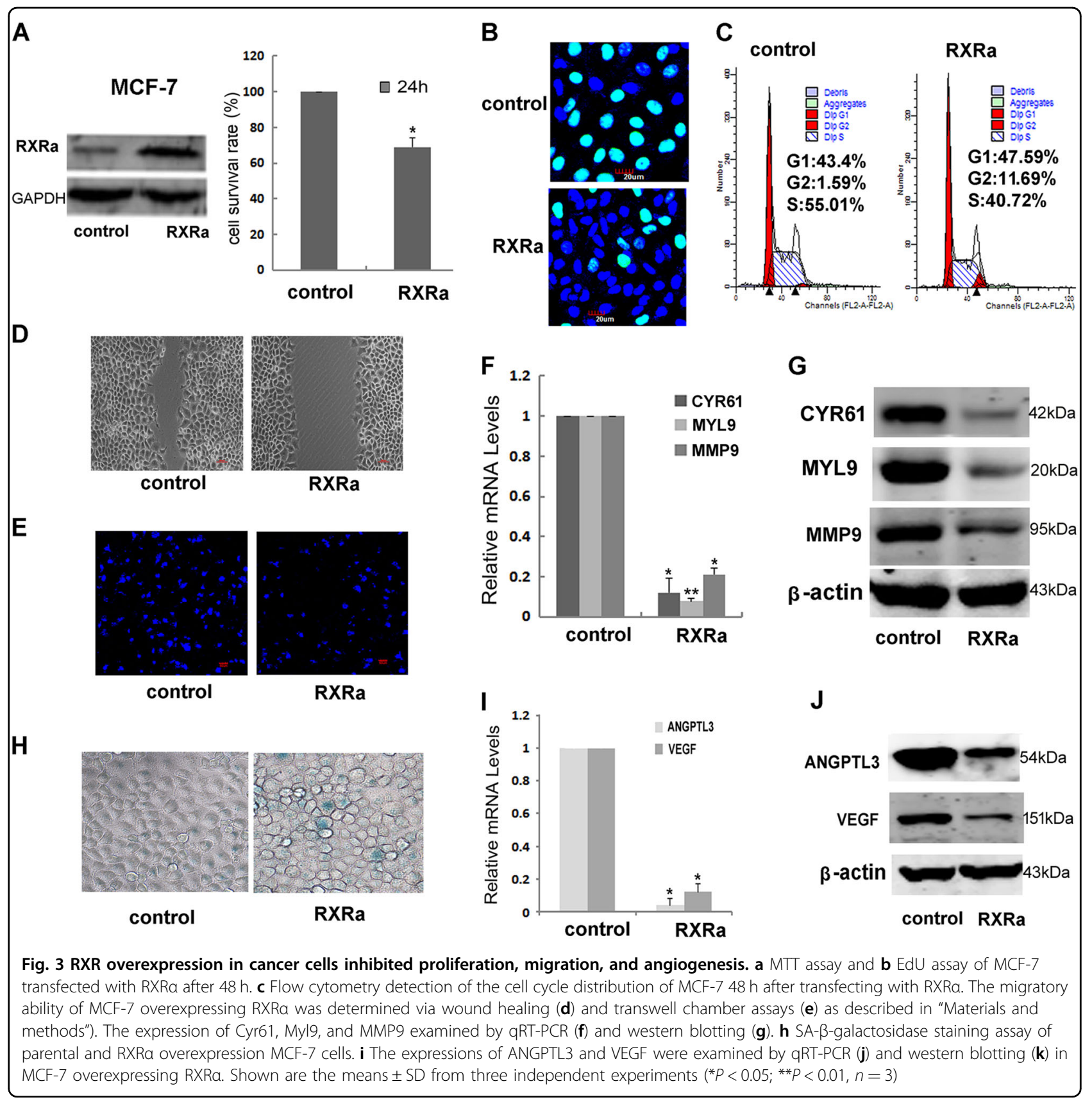

stable overexpression of RXR $\alpha$ in MCF-7 was previously reported to have little change on cell proliferation rates ${ }^{24}$. This discrepancy might be due to some unknown genetic adaptation changes, which need further clarification.

RXR $\alpha$ overexpression also showed strong impacts on cell migration as determined by wound healing ${ }^{25}$ and transwell chamber assays ${ }^{26}$. After creating a "scratch" in a monolayer of MCF-7 cells, the closure of the gap was determined after $24 \mathrm{~h}$. As shown in Fig. 3d, the gap was significantly decreased when cells overexpressing RXR $\alpha$. This was further confirmed by transwell assay. Cells seeded on the upper chamber of a transwell insert crossed the monolayer and migrated to the lower side of the membrane after $24 \mathrm{~h}$ of incubation, which were photographed and counted with confocal microscope. Evident migration and invasion were observed in control MCF-7 cells despite the known fact that this cancer cell line has low invasive potential. When overexpressing RXR $\alpha$ in MCF-7, however, the total number of cells that migrated across the membrane was reduced by more than $40 \%$ (Fig. 3e). Consistently, RXR $\alpha$ overexpression caused a significant reduction of the migration protein markers 
Cyr61, Myl9, and MMP9 in MCF-7 as determined by qRT-PCR and western blot (Fig. $3 \mathrm{f}-\mathrm{g}$ ).

Since the above genes are also known to be altered in senescence. It is interesting to know if senescence was induced in MCF-7 that overexpressing RXR $\alpha$. Therefore, we determined senescence-associated $\beta$-galactosidase (Fig. 3h). Interestingly, the results were different from what we observed in hMSC and there were just some minor increase comparing to mock-treated control MCF7 cell, suggesting that the activation of senescence responses by RXR $\alpha$ is altered in caner cells. Is it due to the ability of cancer cells in sequestrating RXR $\alpha$ to the splicing factor compartments (SFCs) ${ }^{27}$, and therefore leading to the loss of RXR $\alpha$ activity? This is an open question for future studies.

VEGF and the angiopoietin-like family member ANGPTL3 are important players during angiogenesis that are often upregulated by oncogene activation ${ }^{28}$. Consistently, our results showed that the expression of ANGPTL3 and VEGF were both significantly decreased when overexpressing RXR $\alpha$ in MCF-7 (Fig. 3i, j). It was reported that VEGF ligands can be sequestered by the matrix-degrading protease MMP9 leading to a positive feedback regulation ${ }^{29}$. The downregulation of MMP9 expression observed in current study is also in line with that notion. Collectively, the above results revealed that RXR $\alpha$ inhibits angiogenesis, either by itself or through interaction with other cellular components.

\section{RXRa downregulated angiogenesis-related markers partially through interacting with ERa}

RXR $\alpha$ level appears to be tightly regulated in the cell. Although the level of RXR $\alpha$ was elevated together with increasing expression of differentiation markers (as is the case during hMSC differentiation), further increase in the level of RXR $\alpha$ led to suppression of the differentiation markers. This was observed when RXR $\alpha$ was overexpressed in HUVECs. As shown in Figure 4a, three angiogenesis-related marker genes (KDR, eNOs, and EPHB4) were all significantly decreased in HUVECs with RXR $\alpha$ overexpression as compared to that in control HUVECs.

RXRs normally exert their impact through interaction with other nuclear receptors. Noticing the promoter sequence that RXR $\alpha$ recognizes (AGGTCANNNAGG TCA) shares some similarity with that of estrogen receptor $\alpha(\mathrm{ER} \alpha)$ (GGTCANNNTGACCT), we wondered whether RXR $\alpha$ 's participation in regulating angiogenesis has anything to do with ER $\alpha$. Therefore, we investigated the effect of ER $\alpha$ on those differentiation markers in MCF-7 and HeLa, as well as endothelial cells HUVECs, HMVECs, and HAVECs. As shown in Figure 4b, the level of ER $\alpha$ was higher in MCF-7 than that in HUVECs, which is negatively correlated with RXR $\alpha$ (Fig. 1b). To investigate if RXR $\alpha$ and ER $\alpha$ have protein-protein interaction, a co-immunoprecipitation assay was performed. RXR $\alpha$ and ER $\alpha$ expression plasmids were co-transfected in HUVECs. Then, RXR $\alpha$ was immunoprecipitated using anti-RXR $\alpha$ antibody and an immunoblot was carried out with anti-ER $\alpha$ antibody (Fig. 4c, upper panel) and vice versa (Fig. 4c, middle panel). The results showed that RXR $\alpha$ and ER $\alpha$ were co-precipitated from the HUVEC cell extracts in either case, indicating that they do associate with each other. We also found that knockdown of endogenous RXR $\alpha$ by siRNA promoted the expression of ER $\alpha$ as determined by real-time PCR, western blot, and immunocytochemistry (Fig. 4e, f). To further confirm the effect of RXR $\alpha$ on ERa expression, luciferase assay was performed. A reporter construct containing the promoter of ER $\alpha$ was co-transfected with ER $\alpha$ and/or RXR $\alpha$ expression plasmids in COS-7 cells, followed by luciferase measurement. The results showed that RXR $\alpha$ could inhibit the activity of the ER $\alpha$ promoter (Fig. 4g).

To investigate the impacts of $R X R \alpha / E R \alpha$ interaction on cell differentiation, the expression of angiogenesis markers KDR, eNOS, and EphB4 was determined in HUVECs with $\mathrm{RXR} \alpha$ overexpression/knockdown and/or ER $\alpha$ overexpression (Fig. 4). RXR $\alpha$ overexpression strongly inhibited and its knockdown promoted the expression of differentiation markers (Fig. 4a, h). When combined with overexpression of $E R \alpha$, a synergistic effect was observed, suggesting that the impact of RXR $\alpha$ on HUVECs differentiation is (at least partially) through interacting with $E R \alpha$.

\section{Knockdown RXRa rebuilt adult cell's capacity for migration and blood vessel formation, which was enhanced by ERa overexpression}

Cancer cells typically develop alterations that facilitate invasion and metastasis ${ }^{1}$. To check the role of RXR $\alpha$ along this line, we determined its impact on cell migration and blood vessel formation. Migration was assessed by the wound healing assay. As shown in Figure 5a, overexpression of RXR $\alpha$ in hMSCs significantly inhibited cell migration. RXR $\alpha$ overexpression also mildly suppressed the migration capacity of HUVECs and its knockdown promoted migration (Fig. 5b, c). When knockdown of RXR $\alpha$ was combined with ER $\alpha$ overexpression, a significantly elevated cell migration was observed (Fig. 5c). To assess angiogenesis, we performed a Matrigel assay to determine the formation of tube-like structures on an extracellular matrix ${ }^{30}$. Compared to the control HUVECs that formed limited amount of capillary-like structures on Matrigel, RXR $\alpha$ knockdown and/or ER $\alpha$ increased the formation of these structures as evaluated by the total tube area and branching points (Fig. 5d). These results suggest that $\mathrm{RXR} \alpha$, through interacting with $\mathrm{ER} \alpha$, is also involved in controlling the process of cell migration and angiogenesis contributing to cancer cell invasion and metastasis. 

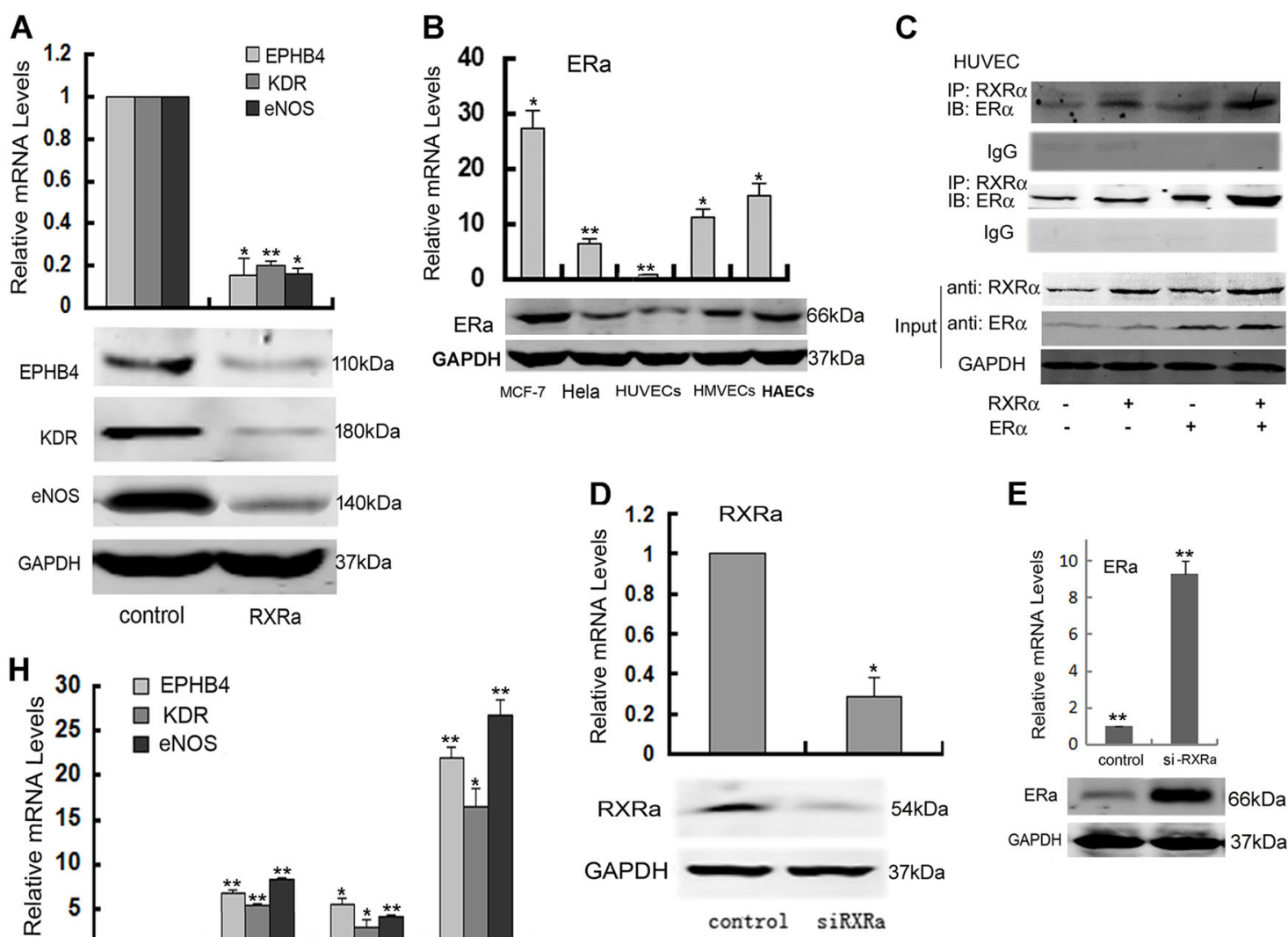

E
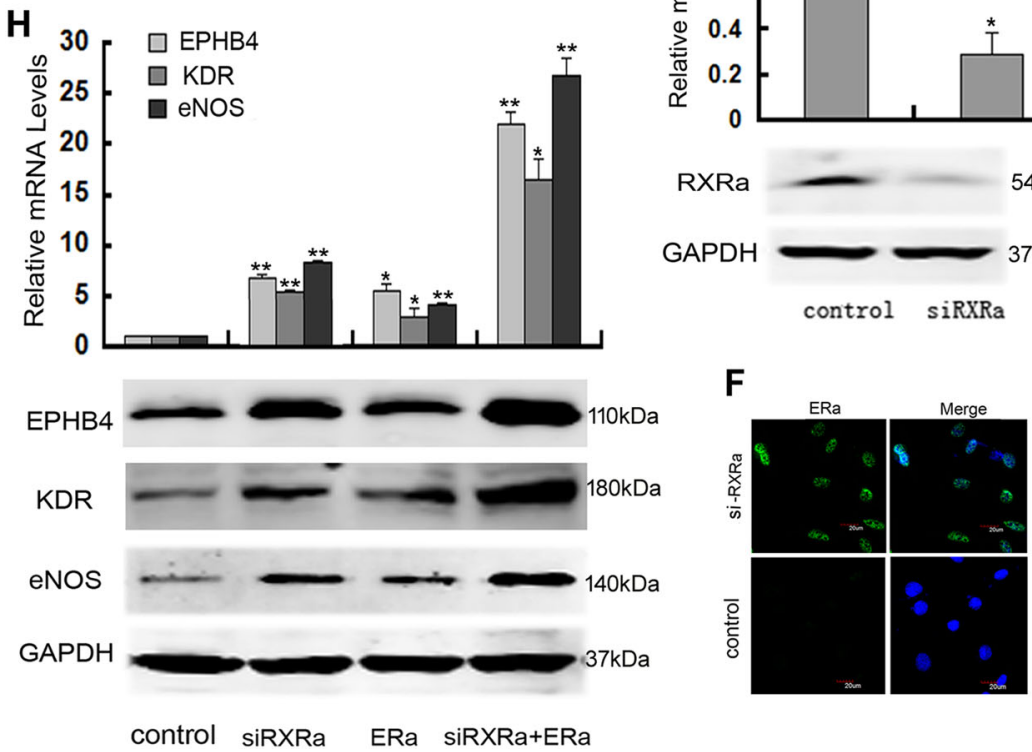

Fig. 4 RXRa downregulated angiogenesis-related makers partially through interacting with ERa. a The expression of EPHB4, KDR, and eNOs in HUVECs overexpressing RXRa. b The expression of ERa in cancer cells MCF-7 and HeLa, endothelial cells HUVECs, HMVECs, and HAVECs. c Co-IP analysis of protein-protein interaction between RXRa and ERa (experiment was performed as described in "Materials and methods"). $\mathbf{d}$ Knockdown of endogenous RXRa in HUVECs with siRXRa. e The expression of ERa examined by qRT-PCR and western blotting in HUVECs following siRXRa knockdown. $\mathbf{f}$ Immunocytochemistry analysis of ERa in HUVECs. $\mathbf{g}$ Binding of RXRa on the promoter of ERa determined by luciferase assay. A plasmid containing the ERa promoter was transfected into COS-7 cells together with plasmids expressing ERa or/and RXRa, and luciferase activities were measured $24 \mathrm{~h}$ later. $\mathbf{h}$ The expression of EPHB4, KDR, and eNOs in HUVECs with siRXRa knockdown or/and ERa overexpression. For the measurements of luciferase reporter and mRNA level, the means \pm SD from three independent experiments are shown $\left({ }^{*} P<0.05 ;{ }^{* *} P<0.01, n=3\right)$

\section{RXRa overexpression caused increased oxygen consumption and metabolism changes}

The growth of cancer cells involves not only a deregulated control of cell proliferation, but also the adjustment of metabolism to provide energy for growth and divi$\operatorname{sion}^{31}$. To check the impacts of RXR $\alpha$ on metabolism reprogramming that might be related to its inhibitory effects on cell differentiation and migration, we investigated the metabolism changes in MCF-7 cell after overexpressing RXR $\alpha$. Glucose, lactate, oxygen consumption, and the expression of metabolic enzymes such as HK2, PFK1, ALDOA, TPI1, PGK1, PGAM1, ENO1, PKM2, and LDHA were measured. As shown in Figure 6a, metabolic change did occur in RXR $\alpha$ overexpressing cells, which showed a lower rate of cellular glucose utilization and lactate production but increased rate of oxygen consumption. Consistently, we found that the mRNA levels of the key enzymes involved in glycolysis, including 


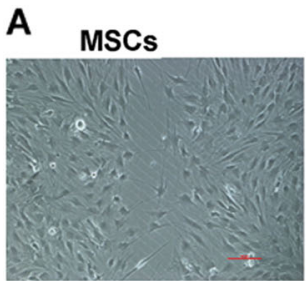

control

C HUVECs

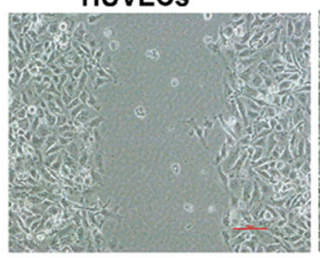

control

D

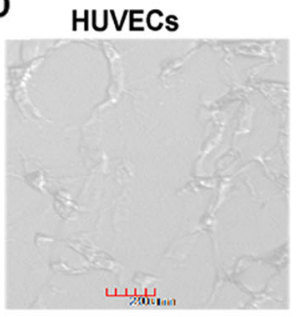

control

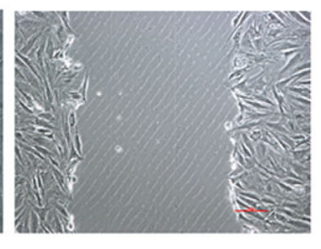

RXRa

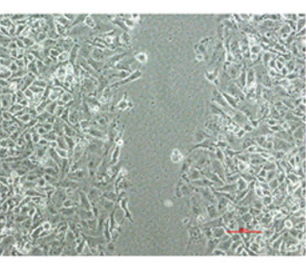

siRXRa

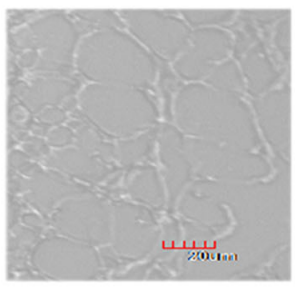

siRXRa
B HUVECS

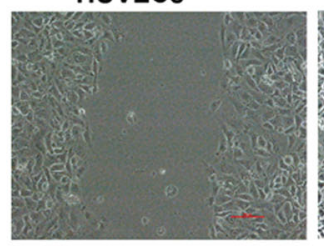

control

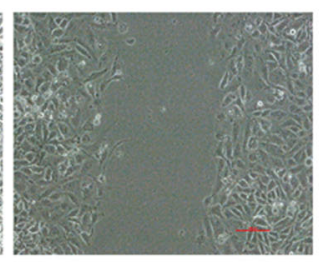

ERa

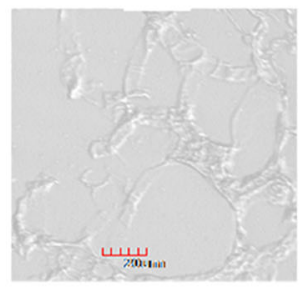

ERa

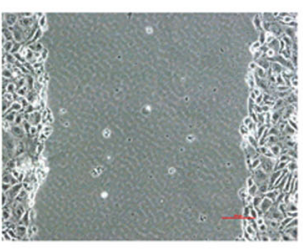

RXRa
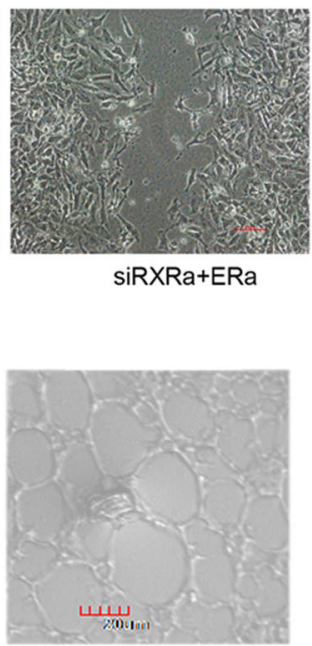

SiRXRa+ERa

Fig. 5 Knockdown of RXR rebuilt adult cell's capacity for migration and blood vessel formation, which was enhanced by ER overexpression. $\mathbf{a}$, b Determination of cell migration by wound healing in hMSCS and HUVECs overexpressing RXRa. c The migration of HUVECS with siRXRa knockdown or/and ERa overexpression. d Blood vessel formation determined by Matrigel assay in HUVECs with siRXRa knockdown or/ and ERa overexpression ( $24 \mathrm{~h}$ culture in Matrigel)

HK2, PKM2, and LDHA, were all decreased in MCF-7 treated with RXR $\alpha$ (Fig. 6b). These results indicated that the RXR $\alpha$ level affects glycolytic processes, as an antagonist for the "aerobic glycolysis" of cancer cells.

\section{Phosphorylation of AKT and FAK contributed to RXRa's impacts on cell differentiation, migration, and angiogenesis}

To understand the downstream signal pathways underlying the diverse inhibitory effects of RXR $\alpha$ on differentiation, angiogenesis, and glycolysis, we determined whether it is related to PI3K/AKT or MAPK pathways, which are well known to transmit multiple cellular signals to influence the above processes ${ }^{32-34}$. As shown in Figure 7a, MCF-7 cells with RXR $\alpha$ overexpression did not change the base level of AKT, but the phosphorylated form of AKT was evidently upregulated. The key component of MAPK pathway-ERK, however, did not show a change on either its base level or the phosphorylated form. These results indicated that RXR $\alpha$ exerted its cellular function through modulation of the PI3K/AKT pathway. We also determined the protein level of the focal adhesion kinase (FAK), which is a cytoplasmic nonreceptor protein-tyrosine kinase that is tightly linked to the embryonic development and tumorigenesis ${ }^{35}$. FAK is known to integrate signals, such as growth factors and mechanical stress, to activate the P13K/Akt and Ras/ MAPK pathway. As shown in Figure 7a, overexpression of RXR $\alpha$ led to the elevation of FAK phosphorylation at Tyr925 (FAK-Y925). This phosphorylated form was reported to reduce cell migration and cell protrusion ${ }^{36}$.

To further confirm that RXR $\alpha$ 's suppression on cell migration was through the AKT pathway, we checked the migration protein markers Cyr61, Myl9, and MMP9 following RXR $\alpha$ overexpression in the presence of AKT inhibitor LY294002, ERK inhibitor PD98059 and the ras homolog gene family member A (RhoA) inhibitor C3. As shown in Figure $7 \mathrm{~b}$, the induction of the three migration protein markers could be fully abrogated by AKT inhibitor LY294002, but not by ERK inhibitor PD98059 or RhoA inhibitor C3.

The involvement of FAK/AKT was also confirmed in HUVEC cells. As shown above in Fig. 4h, the angiogenesis markers KDR, eNOs, and EPHB4 were synergistically 

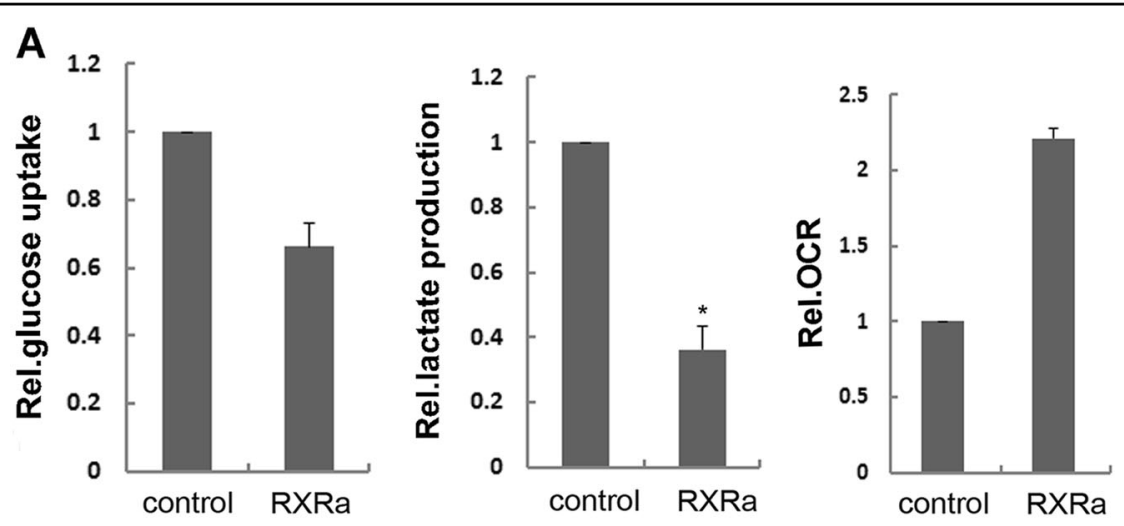

B

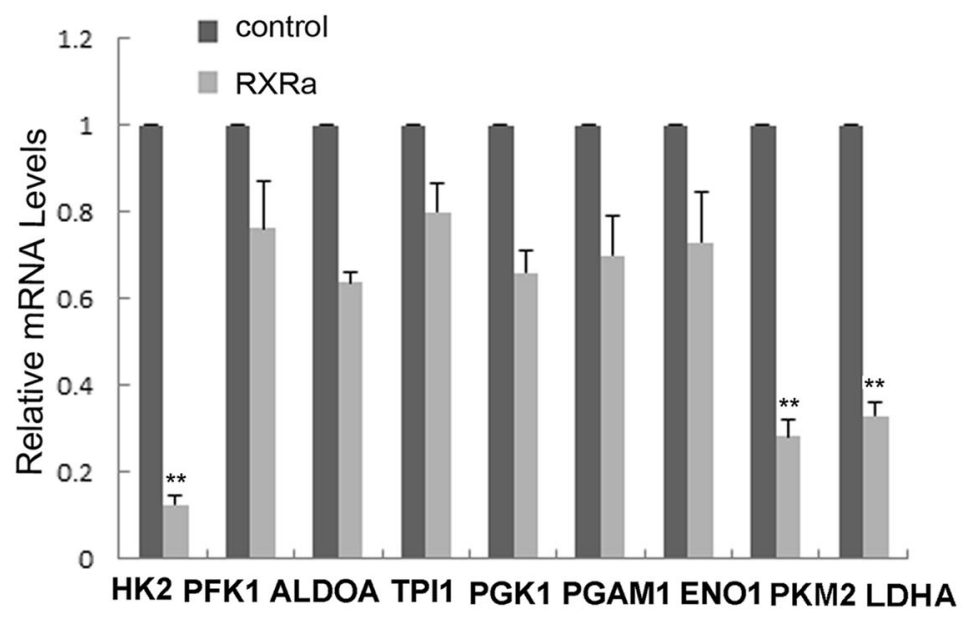

Fig. 6 RXRa overexpression led to increased oxygen consumption and metabolic changes. a Cellular glucose uptake, lactate production, and oxygen consumption rates were measured in MCF-7 overexpressing RXRa using specified assay kit. b qRT-PCR analysis of HK2, PFK1, ALDOA, TPI1, PGK1, PGAM1, ENO1, PKM2, and LDHA in MCF-7 cells overexpressing RXRa

elevated following RXR $\alpha$ knockdown in combination with ER $\alpha$ overexpression. When the same transfected HUVEC cell was pretreated with AKT inhibitor LY294002, the elevation of the three differentiation markers was all efficiently abrogated (Fig. 7c).

Taken together, these results demonstrated that RXR $\alpha$ s inhibitory impacts on angiogenesis and migration were through affecting FAK and AKT pathways via phosphorylation.

\section{Discussion}

Previous studies showed that ligand activations of RXR inhibited the carcinogenic process in leukemia, breast cancer, and other cancers ${ }^{17-19}$, but the underlying mechanism remains elusive. By comparing the impacts of RXR $\alpha$ in cancer cells with those in stem cells on differentiation, angiogenesis, and metabolism, we revealed that RXR $\alpha$ activated multiple cellular events contributing to tumor suppression.

RXR $\alpha$ level appears to be tightly controlled during differentiation. The fact that overexpression of RXR $\alpha$ in
hMSCs did not promote differentiation but activated tumor suppressors (p53, p21, and p16) suggests that activation of RXR $\alpha$ during very early stages of differentiation may lead to harmful consequences, thus triggering the cellular defense mechanism. The senescencelike phenotypes caused by overexpression of RXR $\alpha$ is similar to previous studies with induction of excessive signaling of oncoproteins such as RAS ${ }^{37}$. Interestingly, the senescence-like response seems to occur only in the early stage of stem cell differentiation, overexpression in cancer cells (i.e., MCF-7) did not induce a significant increase of SA- $\beta$-galactosidase. The fact that RXR $\alpha$ can activate the senescence program revealed an important, yet not recognized, mechanism which may contribute to the antitumor effects of RXR $\alpha$ ligands found in clinical trials.

Compared to the elevated levels of RXR $\alpha$ in adult ECs or differentiated hMSCs, the RXR $\alpha$ levels in cancer cells were found to be much lower, similar to that of hMSCs before differentiation (Fig. 1). In connection with the cancer stem cell theory, does this suggest that the cellular mechanism to elevate RXR $\alpha$ be shut down at certain 


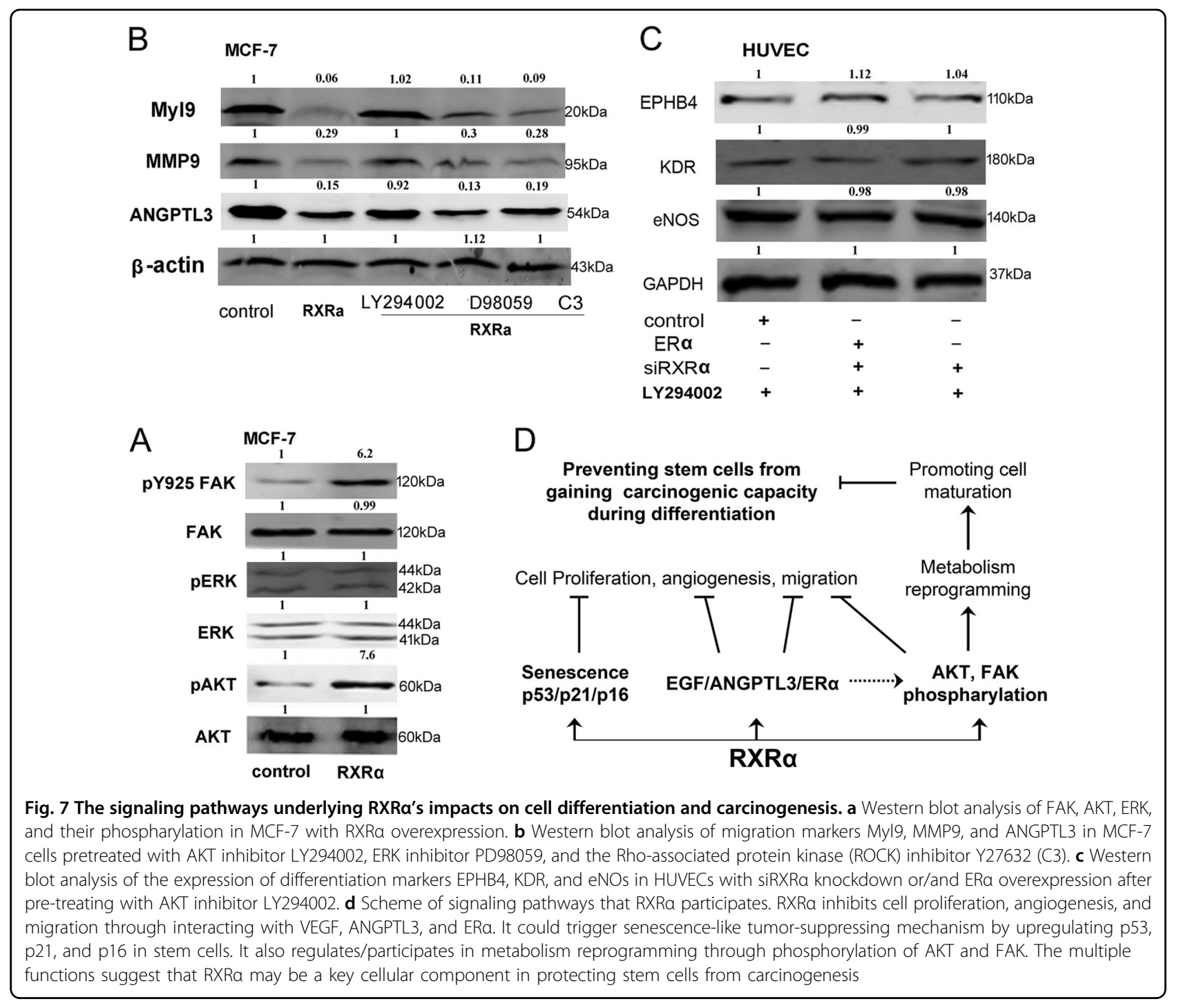

stages during cell differentiation in order for the transformation of stem cells into CSC to occur? Our data support this notion since suppressing RXR $\alpha$ could facilitate carcinogenesis-related features. For example, knockdown of RXR $\alpha$ in normal differentiated cells (HUVECs) could restore their capacity for migration and blood vessel formation (Fig. 5). In cancer cells, overexpressing RXR $\alpha$ not only inhibited cell proliferation, but also migration and angiogenesis (Fig. 3).

Due to its critical function, RXR $\alpha$ deficiency was shown to be embryonic lethal and RXR $\alpha$ expression is rarely lost in human tumors ${ }^{19,38}$. However, the localization of RXR $\alpha$ had been shown to be altered in some cancers by sequestering it to the splicing factor compartments (SFCs), and this caused the loss of its activity ${ }^{27}$. Controlling the level of RXR $\alpha$ or suppressing its activity may be a key requirement during carcinogenesis. Whether this could help drive the transition from stem cell into CSC is an interesting question awaiting further study.

As a hallmark of cancer, the energy production in cancer cells is largely through glycolysis, similar to that of stem cells ${ }^{1}$. It is known that RXR $\alpha$ participates in lipid and glucose metabolism ${ }^{12,13,16}$. Here we demonstrated that RXR $\alpha$ overexpression in cancer cell lines interferes with their metabolism, suppressing enzymes involved in glycolysis which lead to lower rates of glucose utilization and lactate production as well as increased oxygen consumption (Fig. 6). These data revealed another important target of RXR $\alpha$ that is likely underlying its multiple tumor-suppressing functions. During differentiation, a key event occurring in stem cells is the reprogramming of the energy metabolism, shifting from glycolysis toward increased oxidative phosphorylation activity ${ }^{39}$. If cancer cells are originated from normal adult cells, it is difficult 
to understand why they would reprogram their glucose metabolism to glycolysis, which has $\sim 18$-fold lower efficiency for ATP production ${ }^{1}$. But if cancer cells are originated from stem cells, then the progression into cancer cells would not require alteration of the energy metabolism. In this regard, suppressing RXR $\alpha$ to prevent metabolism change is likely a prerequisite for carcinogenesis.

As to the cellular signaling underlying RXRo's multiple impacts on differentiation, carcinogenesis, and metabolism, we found that RXR $\alpha$ 's suppression on angiogenesis appears to be related to its inhibition of VEGF and the angiopoietin-like family member ANGPTL3. Our data also indicated that RXR $\alpha$ 's anti-angiogenic function is at least partly through its interaction with ER $\alpha$, and a synergistic effect was found that impacts differentiation markers. The downstream signal is transmitted via the PI3K-AKT pathway since all the effects of RXR $\alpha$ on cell differentiation, migration, and angiogenesis could be abrogated by inhibiting AKT. We demonstrated that RXR $\alpha$ overexpression caused phosphorylation of AKT and FAK. It has been shown that AKT stimulates histone acetylation that favors proliferation and tumor development, and phosphorylated AKT (S473) correlated with human gliomas and prostate tumors ${ }^{40}$. Phosphorylated FAK at Y925 is most likely responsible for the inhibition of RXR $\alpha$ on cell migration as FAK had been shown to stimulate migration ${ }^{41}$. The fact that RXR $\alpha$-induced phosphorylation of AKT and FAK might as well be related to its inhibition of glycosylase since Akt/PI3K and RAS/FAK signaling correlates with an increase in glucose metabolism ${ }^{42}$.

As illustrated in Fig. 7d, our data revealed the multiple impacts of RXR $\alpha$ - on the one hand, inhibiting cell proliferation, angiogenesis, and migration, as well as promoting tumor-suppressing mechanism (senescence); on the other hand, regulating/participating in differentiation and metabolism reprogramming. These functions, which are at the crossroad between normal cell development and cancer transformation, make RXR $\alpha$ a possible key cellular component that affects the fate of stem cells between specialization/maturation and carcinogenesis.

Collectively, we presented here several lines of evidence showing that RXR $\alpha$ is fine-tuned during stem cell differentiation, and its ectopic expression could trigger multiple signaling pathways on tumor suppression. These results revealed an interesting cross talk between cell differentiation and carcinogenesis.

\section{Materials and methods}

\section{Cell culture and treatment}

Human bone marrow-derived MSCs (hBM-MSCs) were from Union Stem Cell and Gene Engineering Co. The purity of hMSCs was determined by flow cytometry analysis (positive for CD73 and CD105, negative for CD31 and KDR). To induce differentiation toward EC, hMSCs were cultured in differentiation medium (cc-4176, Lonza) supplemented with $50 \mathrm{ng} / \mathrm{mL}$ VEGF (PeproTech), $5 \mathrm{ng} / \mathrm{mL}$ basic fibroblast growth factor (bFGF) (PeproTech), and 2\% fetal bovine serum (FBS) for 7 days. HUVECs were kindly provided by Tianjin Medical University. HMVEC, HAVECs, MCF-7, and Cos-7 were purchased from Shanghai Bio-Tech Co. HUVECs, HMVECs, HAVECs, MCF-7, and Cos-7 were cultured in DMEM/F12 medium supplemented with $10 \%$ fetal bovine serum at $37^{\circ} \mathrm{C}$ in a humidified incubator with a $5 \% \mathrm{CO}_{2}$ atmosphere.

\section{Plasmids, siRNA, and cell transfection}

$E R \alpha$ and RXR $\alpha$ expression plasmids were constructed by subcloning into pcDNA3.1. The primers used for the amplification were listed in supporting information Table 2. For short-term depletion experiments, MSCs, MCF-7, and HUVECs were transfected with siRNA oligonucleotides (supporting information Table 3) using Lipofectamine 2000 (Invitrogen). Transfection reporter assays were performed in 6-well plates. MSCs, HUVECs, and MCF-7 cells were cultured in growth medium without antibiotics at $60 \%$ confluence for 2 days. MSCs were then transfected using the FuGENEHD transfection reagent (Roche); HUVECs and MCF-7 cells were transfected using the TurboFect transfection reagent (Thermo). After $48 \mathrm{~h}$, cells were used for testing at mRNA and protein levels.

\section{MTT assay and EdU cell proliferation detection}

Cell viability was examined by 3-(4,5-dimethylthiazol-2yl)-2,5-diphenyltetrazolium (MTT) assay (Sigma). The absorbance of each well was measured using a Synergy ${ }^{\text {tix }} 4$ plate reader (Bioteck) with a test wavelength at $490 \mathrm{~nm}$ and a reference wavelength set at $630 \mathrm{~nm}$. Absorbance is directly proportional to the number of survival cells.

EdU staining was conducted using Cell-Light EdU Apollo 488 in vitro kit (Ribobio), according to the manufacturer's protocol. A total of $1 \times 10^{5}$ cells were incubated with $50 \mu \mathrm{M}$ EdU for $2 \mathrm{~h}$. After fixation and permeabilization, the incorporated EdU was visualized by means of a click reaction using Alexa Fluor 488 azide (30 min, room temperature (RT)). The nuclear DNA was stained with DAPI ( $30 \mathrm{~min}, \mathrm{RT})$. The images were observed using confocal laser scanning microscope, and quantification was performed by counting the positively stained cells in five randomly selected areas under microscope.

\section{In vitro angiogenesis assay}

Capillary tube formation of HUVECs and MCF-7 was induced using basement membrane-like material (EC Matrix TM; BD) as previously described ${ }^{43}$. Briefly, basement membrane-like material was diluted to $0.5-0.7 \mathrm{mg} /$ $\mathrm{mL}$ in DMEM/F12 medium. A total of $5 \times 10^{4}$ cells were 
seeded in $300 \mu \mathrm{L}$ of $0.5-0.7 \mathrm{mg} / \mathrm{mL}$ Matrigel in each well of a 24-well plate. The Matrigel cell suspension was polymerized for $4 \mathrm{~h}$ at $37^{\circ} \mathrm{C}$. Then, $300 \mu \mathrm{L}$ of DMEM/F12 medium supplemented with $50 \mathrm{ng} / \mathrm{mL}$ VEGF was added, and the gel-embedded cells were cultured at $37{ }^{\circ} \mathrm{C}$ and $4 \%$ $\mathrm{CO}_{2}$. The structures were photographed using a phase contrast microscope (Olympus) after $48 \mathrm{~h}$. Total cord length was quantified using image-Pro Plus v4.5 software.

\section{Cell migration assay}

MSCs, HUVECs, and MCF-7 grown in 6-well plates were transfected with RXR $\alpha$ and then wounded using a sterile pipette tip as described in ref. ${ }^{25}$. The progress of migration was photographed immediately following injury, and micrographs were taken at 0,24 , and $48 \mathrm{~h}$.

\section{Transwell chamber assay}

After MCF-7 cells were transfected with RXR $\alpha$, cells were harvested by trypsin, and $1.0 \times 10^{4}$ cells in $200 \mu \mathrm{L}$ of $1 \%$ FBS-containing medium were then seeded into the upper chamber of a transwell cell culture insert as previously described ${ }^{26}$. The lower chamber was filled with $600 \mu \mathrm{L}$ of medium containing 10\% FBS. Twenty-four hours later, the cells in the upper chamber were removed using a cotton swab. Cells that had migrated to the lower side of the membrane were fixed with $4 \%$ paraformaldehyde and stained with DAPI. The number of migrated cells was counted and photographed in five fields (the upper, lower, left, right, and middle) under microscope, and the average number was obtained from three independent experiments.

\section{Detection of senescence-associated $\beta$-galactosidase}

SA- $\beta$-gal was detected according to Dimri et al. ${ }^{22,}{ }^{23}$. After hMSCs transfected with RXR $\alpha$ for $48 \mathrm{~h}$, cells were fixed with $4 \%$ formaldehyde, washed and exposed overnight at $37^{\circ} \mathrm{C}$ to a solution containing $1 \mathrm{mg} / \mathrm{mL} 5$-bromo4-chloro-3-indolyl- $\beta$-galactopyranoside, $5 \mathrm{mM}$ potassium ferrocyanide, $5 \mathrm{mM}$ potassium ferricyanide, $150 \mathrm{mM}$ $\mathrm{NaCl}, 2 \mathrm{mM} \mathrm{MgCl} 2$, and $0.1 \mathrm{M}$ phosphate buffer, $\mathrm{pH}$ 6.0. Then, the staining was photographed using a phase contrast microscope (Olympus).

\section{Quantitative real-time RT-PCR (qRT-PCR)}

Total RNA was isolated from cells using Trizol reagent (Invitrogen), reverse-transcribed complementary DNA was synthesized with random primers or microRNAs specific stem-loop primers. qRT-PCR analysis was performed using Fast SYBR Green Master Mix (Applied Biosystems) in a Biosystems StepOneTM Real-Time PCR machine (Applied Biosystem, CA). Glyceraldehyde-3phosphate dehydrogenase (GAPDH) was used as internal controls. The primers used for qRT-PCR were listed in supporting information Table 1.

\section{Western blotting}

After lysing cells with extraction buffer, cell extracts were separated by SDS-PAGE and then transferred onto nitrocellulose membranes. The following primary antibodies were used: rabbit anti-eNOS, KDR, Cyr61, Myl9, MMP9, ANGPTL3, and VEGF (abcam); EPHB4, RXR $\alpha$, ER $\alpha$, pAKT, AKT, pY925 FAK, FAK, pERK, ERK, and mouse anti-GAPDH (Santa Cruz Biotechnology). Antibody incubations were performed overnight at $4{ }^{\circ} \mathrm{C}$. The secondary antibodies used were IRDyeTM-800-conjugated antimouse and anti-rabbit IgG (Li-COR Biosciences). Immunoreactivity was detected using an Odyssey Infrared Imaging System (Gene Company Limited). All immunoblots were repeated at least two-three times.

\section{Immunoprecipitation (IP) analysis}

HUVEC cells were co-transfected with RXR $\alpha$ and ER $\alpha$. Protein extracts were isolated from cells using RIPA buffer. Tagged proteins were immunoprecipitated overnight at $4{ }^{\circ} \mathrm{C}$ using protein $\mathrm{A} / \mathrm{G}$ agarose (CW0349) and anti-RXR $\alpha$ antibody, and then all complexes were pelleted at $3000 \mathrm{rpm}$ for $3 \mathrm{~min}$. The beads were washed and fractionated by $12 \%$ SDS-PAGE, followed by transfer to a nitrocellulose membrane. The membrane was immunoblotted with mouse anti-ER $\alpha$ (1:5000) and $\beta$-actin (1:250) overnight at $4{ }^{\circ} \mathrm{C}$ and then incubated with anti-mouse secondary antibodies (Li-COR Biosciences) for $1 \mathrm{~h}$ at RT. The specific proteins were visualized by Odyssey Infrared Imaging System (Gene Company Limited).

\section{Immunocytochemistry}

The cells were fixed in 4\% paraformaldehyde for $15 \mathrm{~min}$, then blocked with normal goat serum for $20 \mathrm{~min}$ at room temperature. After incubation with rabbit anti-RXR $\alpha$ (santa cruz) and ERa (santa cruz) in a humid chamber overnight, cells were incubated with appropriate secondary antibodies (fluorescein isothiocyanate (FITC)-labeled goat anti-rabbit IgG) for $30 \mathrm{~min}$ at $37^{\circ} \mathrm{C}$. Washing with $\mathrm{PBS}$, and then the samples were observed under laser scanning confocal microscope (OLYMPUS). DAPI stain (blue) high lights the total nuclei.

\section{Luciferase assay}

Luciferase activity assay was performed using the Luciferase Assay System (Promega) according to the instructions. Briefly, HUVEC cells were seeded in 24-well plates, ERa reporter plasmids (ERa-2235-Luc) were cotransfected with sh-RXR $\alpha$ plasmids or control (pSUPER) plasmids using Lipofectamine 2000 for $24 \mathrm{~h}$. ERa promoter plasmids and RXR $\alpha$ expression plasmids and/or ERa expression plasmids were co-transfected into COS-7 cells using Lipofectamine 2000 for $24 \mathrm{~h}$. The transfected cells were lysed in Cell Culture Lysis Reagent. An aliquot of $20 \mu \mathrm{L}$ of cell lysate was added into a 96-well enzyme 
label plate and reading was initiated by the injection of $100 \mu \mathrm{L}$ of Luciferase Assay Reagent into the plate on a Synergy $^{\text {tw }} 4$ (Bioteck). Transfection efficiencies were normalized by total protein concentration of each luciferase assay preparation.

\section{Statistical analysis}

Data were expressed as the mean $\pm \mathrm{SE}$, accompanied by the number of experiments performed independently, and analyzed by $t$-test. Differences with $P<0.05$ were considered statistically significant.

\section{Acknowledgements}

We thank James Westmoreland and Daniel Menendez for proofreading of the manuscript and critical suggestions. This work was supported by The National Key R\&D Program of China (2017YFD0400304) and the Innovative Research Team of Tianjin Municipal Education Commission (TD13-5015).

\section{Author details}

${ }^{1}$ College of Biotechnology, Tianjin University of Science and Technology, 300457 Tianjin, China. ${ }^{2}$ Institute of Biology and Medicine, Wuhan University of Science and Technology, 430081 Wuhan, China. ${ }^{3}$ Qilu Institute of Technology, 250200 Shandong, China

\section{Author contributions}

R.Z., H.L., and S.Z. performed the experiments and analysis of data. W.M. contributed to the concepts and wrote the manuscript. T.-C.Z. contributed to the design of the study. Y.Z. and N.W. participated in parts of the experiments. H.Z. prepared the materials. H.H. and G.H. contributed to technical guidance and support. All authors read and approved the final manuscript.

\section{Conflict of interest}

The authors declare that they have no conflict of interest.

\section{Publisher's note}

Springer Nature remains neutral with regard to jurisdictional claims in published maps and institutional affiliations.

Supplementary Information accompanies this paper at (https://doi.org/ 10.1038/s41419-018-0610-1).

Received: 29 September 2017 Revised: 31 March 2018 Accepted: 10 April 2018

Published online: 10 May 2018

\section{References}

1. Hanahan, D. \& Weinberg, R. A. Hallmarks of cancer: the next generation. Cell 144, 646-674 (2011).

2. López-Lázaro, M. Stem cell division theory of cancer. Cell Cycle 14, 2547-2548 (2015).

3. Smalley, M. \& Ashworth, A. Stem cells and breast cancer: a field in transit. Nat. Rev. Cancer 3, 832-844 (2003).

4. Al-Hajj, M., Wicha, M. S., Benito-Hernandez, A., Morrison, S. J. \& Clarke, M. F. Prospective identification of tumorigenic breast cancer cells. Proc. Natl. Acad. Sci. USA 100, 3983-3988 (2003).

5. Bonnet, D. \& Dick, J. E. Human acute myeloid leukemia is organized as a hierarchy that originates from a primitive hematopoietic cell. Nat. Med. $\mathbf{3}$ 730-737 (1997).

6. Creighton, C. J. et al. Residual breast cancers after conventional therapy display mesenchymal as well as tumor-initiating features. Proc. Natl. Acad. Sci. USA 106, 13820-13825 (2009).

7. Kim, C. F. B. et al. Identification of bronchioalveolar stem cells in normal lung and lung cancer. Cell 121, 823-835 (2005).
8. Dawood, S., Austin, L. \& Cristofanilli, M. Cancer stem cells: implications for cancer therapy. Oncol. Williston Park N. 28, 1101-1107 (2014).

9. López-Lázaro, M. Cancer arises from stem cells: opportunities for anticancer drug discovery. Drug Discov. Today 20, 1285-1287 (2015).

10. Singh, A. \& Settleman, J. EMT, cancer stem cells and drug resistance: an emerging axis of evil in the war on cancer. Oncogene 29,4741-4751 (2010).

11. Tomasetti, C. \& Vogelstein, B. Variation in cancer risk among tissues can be explained by the number of stem cell divisions. Science 347, 78-81 (2015).

12. Altucci, L., Leibowitz, M. D., Ogilvie, K. M., de Lera, A. R. \& Gronemeyer, H. RAR and RXR modulation in cancer and metabolic disease. Nat. Rev. Drug Discov. 6 , 793-810 (2007).

13. Nagy, L., Szanto, A., Szatmari, I. \& Széles, L. Nuclear hormone receptors enable macrophages and dendritic cells to sense their lipid environment and shape their immune response. Physiol. Rev. 92, 739-789 (2012).

14. Wang, W. et al. Rapid and efficient reprogramming of somatic cells to induced pluripotent stem cells by retinoic acid receptor gamma and liver receptor homolog 1. Proc. Natl. Acad. Sci. USA 108, 18283-18288 (2011).

15. Yang, J. et al. Signalling through retinoic acid receptors is required for reprogramming of both mouse embryonic fibroblast cells and epiblast stem cells to induced pluripotent stem cells. Stem Cells Dayt. Ohio 33, 1390-1404 (2015).

16. Lefebvre, P., Benomar, Y. \& Staels, B. Retinoid X receptors: common heterodimerization partners with distinct functions. Trends Endocrinol. Metab. TEM 21, 676-683 (2010).

17. Bunaciu, R. P. \& Yen, A. Activation of the aryl hydrocarbon receptor AhR promotes retinoic acid-induced differentiation of myeloblastic leukemia cells by restricting expression of the stem cell transcription factor Oct4. Cancer Res. 71, 2371-2380 (2011).

18. Crowe, D. L. \& Chandraratna, R. A. S. A retinoid X receptor (RXR)-selective retinoid reveals that $\mathrm{RXR}$-alpha is potentially a therapeutic target in breast cancer cell lines, and that it potentiates antiproliferative and apoptotic responses to peroxisome proliferator-activated receptor ligands. Breast Cancer Res. BCR 6, R546-R555 (2004).

19. Tanaka, T. \& De Luca, L. M. Therapeutic potential of 'rexinoids' in cancer prevention and treatment. Cancer Res. 69, 4945-4947 (2009).

20. Hanahan, D. \& Folkman, J. Patterns and emerging mechanisms of the angiogenic switch during tumorigenesis. Cell 86, 353-364 (1996).

21. Ball, S. G., Shuttleworth, C. A. \& Kielty, C. M. Platelet-derived growth factor receptors regulate mesenchymal stem cell fate: implications for neovascularization. Expert Opin. Biol. Ther. 10, 57-71 (2010).

22. Dimri, G. P. et al. A biomarker that identifies senescent human cells in culture and in aging skin in vivo. Proc. Natl. Acad. Sci. USA 92, 9363-9367 (1995).

23. Debacq-Chainiaux, F., Erusalimsky, J. D., Campisi, J. \& Toussaint, O. Protocols to detect senescence-associated beta-galactosidase (SA-ßgal) activity, a biomarker of senescent cells in culture and in vivo. Nat. Protoc. 4, 1798-1806 (2009).

24. Crowe, D. L. \& Chandraratna, R. A. S. A retinoid X receptor (RXR)-selective retinoid reveals that $R X R$-alpha is potentially a therapeutic target in breast cancer cell lines, and that it potentiates antiproliferative and apoptotic responses to peroxisome proliferator-activated receptor ligands. Breast Cancer Res. BCR 6, R546-R555 (2004).

25. Liang, C.-C., Park, A. Y. \& Guan, J.-L. In vitro scratch assay: a convenient and inexpensive method for analysis of cell migration in vitro. Nat. Protoc. 2, 329-333 (2007).

26. Marshall, J. Transwell( $\left({ }^{\circ}\right)$ invasion assays. Methods Mol. Biol. Clifton NJ 769 , 97-110 (2011).

27. Tanaka, T. et al. Altered localization of retinoid $X$ receptor alpha coincides with loss of retinoid responsiveness in human breast cancer MDA-MB-231 cells. Mol. Cell. Biol. 24, 3972-3982 (2004).

28. Ferrara, N. Pathways mediating VEGF-independent tumor angiogenesis. Cytokine Growth Factor Rev. 21, 21-26 (2010).

29. Kessenbrock, K., Plaks, V. \& Werb, Z. Matrix metalloproteinases: regulators of the tumor microenvironment. Cell 141, 52-67 (2010).

30. Ponce, M. L. Tube formation: an in vitro matrigel angiogenesis assay. Methods Mol. Biol. Clifton NJ 467, 183-188 (2009).

31. Jones, R. G. \& Thompson, C. B. Tumor suppressors and cell metabolism: a recipe for cancer growth. Genes Dev. 23, 537-548 (2009).

32. Manning, B. D. \& Cantley, L. C. AKT/PKB signaling: navigating downstream. Cell 129, 1261-1274 (2007).

33. Elstrom, R. L. et al. Akt stimulates aerobic glycolysis in cancer cells. Cancer Res. 64, 3892-3899 (2004). 
34. Mebratu, Y. \& Tesfaigzi, Y. How ERK1/2 activation controls cell proliferation and cell death: is subcellular localization the answer? Cell Cycle 8, 1168-1175 (2009).

35. Sulzmaier, F. J., Jean, C. \& Schlaepfer, D. D. FAK in cancer: mechanistic findings and clinical applications. Nat. Rev. Cancer 14, 598-610 (2014).

36. Deramaudt, T. B. et al. FAK phosphorylation at Tyr-925 regulates cross-talk between focal adhesion turnover and cell protrusion. Mol. Biol. Cell 22 964-975 (2011).

37. Serrano, M., Lin, A. W., McCurrach, M. E., Beach, D. \& Lowe, S. W. Oncogenic ras provokes premature cell senescence associated with accumulation of p53 and p16INK4a. Cell 88, 593-602 (1997).

38. Dyson, E. et al. Atrial-like phenotype is associated with embryonic ventricular failure in retinoid X receptor alpha -/- mice. Proc. Natl. Acad. Sci. USA 92, 7386-7390 (1995).
39. Teslaa, T. \& Teitell, M. A. Pluripotent stem cell energy metabolism: an update EMBO J. 34, 138-153 (2015).

40. Lee, J. V. et al. Akt-dependent metabolic reprogramming regulates tumor cell histone acetylation. Cell Metab. 20, 306-319 (2014)

41. Sieg, D. J. et al. FAK integrates growth-factor and integrin signals to promote cell migration. Nat. Cell Biol. 2, 249-256 (2000).

42. Nicholson, K. M. \& Anderson, N. G. The protein kinase B/Akt signalling pathway in human malignancy. Cell Signal. 14, 381-395 (2002).

43. Donovan, D., Brown, N. J., Bishop, E. T. \& Lewis, C. E. Comparison of three in vitro human 'angiogenesis' assays with capillaries formed in vivo. Angiogenesis 4, 113-121 (2001). 